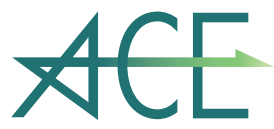

Annals of Clinical Epidemiology

\title{
Status of Anemia According to Underlying Renal Disease in Chronic Kidney Disease: The Fukushima CKD Cohort
}

\author{
Kenichi Tanaka ${ }^{1,2}$, Hirotaka Saito ${ }^{1}$, Tsuyoshi Iwasaki ${ }^{1}$, Akira Oda ${ }^{1}$, Shuhei Watanabe ${ }^{1}$, \\ Makoto Kanno ${ }^{1,2}$, Hiroshi Kimura ${ }^{1}$, Michio Shimabukuro ${ }^{2,3}$, Koichi Asahi' ${ }^{4}$, Tsuyoshi Watanabe ${ }^{2}$, \\ Junichiro James Kazama ${ }^{1,2}$
}

\section{ABSTRACT}

BACKGROUND

Although anemia is an important factor determining renal and cardiovascular outcomes in patients with chronic kidney disease (CKD), reports on the status of anemia management in Japanese patients with pre-dialysis CKD are limited.

METHODS

We retrospectively analyzed CKD patients follow-up at our department between June 2012 and July 2014 (Fukushima CKD cohort study), for the management status of anemia and differences in anemia control under the current treatment strategy, including long-acting erythropoiesis stimulating agents (ESA), classified according to the underlying renal disease, namely primary renal disease, hypertensive nephropathy, diabetic nephropathy, and others. Anemia was defined as a hemoglobin level of $<11 \mathrm{~g} / \mathrm{dL}$, or receiving ESA therapy.

RESULTS

We identified 1,324 patients with CKD, and found that the prevalence of anemia increased with CKD stage, and was significantly higher in diabetic nephropathy than primary renal disease and hypertensive nephropathy $(25.3 \%, 12.9 \%$, and $10.9 \%$, respectively, $P<0.001)$. Anemia was independently related to the underlying renal disease, with a significant difference between diabetic nephropathy and primary renal disease (odds ratio 2.15 ; $95 \%$ confidence interval, 1.16-3.99, $P=0.015)$. Among those with hemoglobin $<11 \mathrm{~g} / \mathrm{dL}, 57.1 \%$ of patients were not on ESAs. The mean hemoglobin level was $10.4 \pm 1.0 \mathrm{~g} / \mathrm{dL}$ and achievement rate of a target hemoglobin of $\geq 11 \mathrm{~g} / \mathrm{dL}$, recommended in Japanese guidelines, was $30.1 \%$ among subjects on ESA therapy.

CONCLUSIONS

These results suggest that intervention for renal anemia is not necessarily adequate among Japanese patients with CKD, even those under nephrologist care, including with use of longacting ESAs.

KEY WORDS

Anemia, Chronic kidney disease, Underlying renal disease, Diabetic nephropathy

\author{
${ }^{1}$ Department of Nephrology and Hyperten- \\ sion, Fukushima Medical University \\ 2 Department of Chronic Kidney Disease \\ Initiatives, Fukushima Medical University \\ ${ }^{3}$ Department of Diabetes, Endocrinology \\ and Metabolism, Fukushima Medical Uni- \\ versity \\ ${ }^{4}$ Division of Nephrology and Hypertension, \\ Iwate Medical University \\ Corresponding author: Kenichi Tanaka \\ Department of Nephrology and Hyperten- \\ sion, Fukushima Medical University, 1, \\ Hikarigaoka, Fukushima City, Fukushima \\ 960-1295, Japan \\ E-mail: kennichi@fmu.ac.jp \\ Received: September 11, 2020 \\ Accepted: November 18, 2020 \\ No.21-04 \\ (C) 2021 Society for Clinical Epidemiology
}




\section{INTRODUCTION}

hronic kidney disease (CKD), an independent risk factor for cardiovascular disease, all-cause mortality and end-stage renal disease (ESRD) in the general population $[1,2]$, has become a worldwide public health burden. Globally, measures to prevent the development and progression of CKD are urgently required. The proportion of patients with $\mathrm{CKD}$ is reportedly higher in Japan than in foreign countries, with an estimated number of CKD patients in Japan of 13.3 million (corresponding to $13 \%$ of the adult population) [3]. Japan is one of the countries with the highest incidence of ESRD, and in fact, the number of Japanese patients with ESRD has increased over the past four decades [4]. CKD is thus a major public health and economic burden in Japan.

Various factors are associated with the development and progression of $\mathrm{CKD}$, including age, hypertension, diabetes mellitus, dyslipidemia, obesity, smoking, proteinuria and hematuria [5]. Anemia is common in patients with CKD and its prevalence increases with CKD stage advancement [6]. Although renal anemia is a modifiable risk factor that affects both the renal and cardiovascular outcomes of CKD patients [1,7], only a limited number of reports have examined the status of anemia management in Japanese patients with pre-dialysis CKD. Akizawa et al. reported on the prevalence of anemia and the practice of treatment with erythropoiesis stimulating agents (ESA) using baseline data of the Chronic Kidney Disease Japan Cohort (CKD-JAC) study, a cohort of pre-dialysis CKD patients in Japan, and revealed that a large number of patients receiving ESAs failed to maintain the recommended hemoglobin concentration [8]. The ESA used in the CKD-JAC study was only epoetin alfa and beta, administered once every two weeks, and the difficulty in maintaining appropriate dosing intervals in outpatient settings was thought to have contributed to the poor management of anemia in their study. Although longacting ESAs, administered once a month, could be superior to short-acting ESAs in terms of maintaining an adequate hemoglobin concentration in pre-dialysis patients with $\mathrm{CKD}$, reports on the management status of renal anemia in subjects on long-acting ESAs are limited. Moreover, the relationships between anemia control, CKD stage, underlying renal disease and ESA treatment were not sufficiently evaluated in pre-dialysis CKD patients under nephrologists' care, despite the fact that the risks of cardiovascular disease and mortality due to CKD need to be stratified according to both the underly- ing renal disease and CKD stage [9]. Therefore, the aim of the present study was to investigate the prevalence and management status of anemia and its related factors, in particular, the underlying renal disease, in Japanese patients with pre-dialysis CKD under the current treatment strategy, which includes the long-acting ESAs, darbepoetin and epoetin beta pegol.

\section{METHODS}

\section{STUDY POPULATION (FUKUSHIMA CKD COHORT)}

The Fukushima CKD Cohort Study is a prospective survey to investigate the characteristics and outcomes, such as cardiovascular events, ESRD and death, of pre-dialysis patients with CKD at Fukushima Medical University Hospital [10]. The study was registered in the University Hospital Medical Information Network Clinical Trials Registry (UMIN-CTR) UMIN000040848. The present study was a cross-sectional survey conducted using the baseline data of the Fukushima CKD cohort study.

The inclusion criteria were as follows: (1) Japanese patients living in Japan; (2) age 18 years or over; and (3) presence of CKD according to the definition of an estimated GFR of $<60 \mathrm{~mL} / \mathrm{min} / 1.73 \mathrm{~m}^{2}$ or positive dipstick results for proteinuria $(\geq 1+)$, with stable renal function for at least three months before entry into the study. eGFR was calculated using the estimation equation for Japanese patients with CKD. This equation calculates the eGFR from serum creatinine, age and sex using the following formula: $\left(\mathrm{eGFR}\left[\mathrm{mL} / \mathrm{min} / 1.73 \mathrm{~m}^{2}\right]=194 \times\right.$ age $^{-0.287} \times$ serum creatinine $e^{-1.094} \times[0.739$ for women $\left.]\right)$ [11]. The exclusion criteria were as follows: (1) dialysis treatment in the last three months, (2) active malignancy, (3) infectious disease, (4) pregnancy, and (5) history of organ transplantation. Recruitment commenced in June 2012, and 1,479 patients were initially recruited. Patients with active malignancy, infectious diseases, or pregnancy were not recruited at this time as determined by their attending physician. Of the originally registered patients, those lacking data on serum creatinine were excluded from this analysis. All patients were under the care of specialists in nephrology or diabetology. The protocol was approved by the ethics committee of Fukushima Medical University and the study was conducted in accordance with the Declaration of Helsinki. All patients provided written, informed consent for participation.

\section{PATIENT CLASSIFICATION}

Patients were classified according to the presence of one of four underlying renal diseases, as diagnosed by their 
attending physicians: primary renal disease, defined as primary glomerulonephritis including cases not proven by biopsy; hypertensive nephropathy, defined by a preceding history of hypertension with absence of other possible disorders, including cases with biopsy findings of nephrosclerosis; diabetic nephropathy, defined by a preceding history of diabetes mellitus with accompanying nephropathy without other possible renal disorders, including cases with biopsy findings of diabetic nephropathy or those presenting with nephropathy along with diabetic retinopathy in the absence of other possible disorders; other nephropathies, defined as other nephropathies not included in the other three groups (systemic vasculitis, collagen diseases, tubulointerstitial nephritis, drugs, vascular disorders, urological disorders, genetic disorders, and unknown).

\section{MEASUREMENTS}

Information on medications at baseline, as well as history of cardiovascular disease, diabetes mellitus, hypertension and dyslipidemia, were obtained from the patients' medical records or from the results of blood examinations at registration. Systolic and diastolic blood pressure were measured by trained staff using a standard sphygmomanometer or an automated device with the subjects in the sitting position. Hypertension was defined as a systolic blood pressure $\geq 140 \mathrm{~mm} \mathrm{Hg}$, diastolic blood pressure $\geq 90 \mathrm{~mm} \mathrm{Hg}$, or use of antihypertensive medication. Body mass index was calculated as weight $(\mathrm{kg})$ divided by height squared (in meters, $\mathrm{m}^{2}$ ). Subjects with diabetes were identified by a fasting plasma glucose concentration $\geq 126 \mathrm{mg} / \mathrm{dL}$ or a glycosylated hemoglobin (HbA1c) value (National Glycohemoglobin Standardization Program) $\geq 6.5 \%$, or those who used insulin or oral antihyperglycemic drugs. Dyslipidemia was defined as a triglyceride concentration $\geq 150 \mathrm{mg} / \mathrm{dL}$, or low-density lipoprotein cholesterol concentration $\geq 140 \mathrm{mg} / \mathrm{dL}$, or high-density lipoprotein cholesterol concentration $<40 \mathrm{mg} / \mathrm{dL}$, or use of antihyperlipidemic medication. Cardiovascular diseases included myocardial infarction, angina pectoris, congestive heart failure, arrhythmias, cerebrovascular disorders, chronic arteriosclerosis obliterans and aortic dissection. Anemia was defined as a hemoglobin level of $<11 \mathrm{~g} / \mathrm{dL}$, or receiving ESA therapy, as described in a previous study [8]. Almost all ESAs used in the present study were long-acting ESAs; darbepoetin was used in 55 patients, epoetin beta pegol in 41 patients, and epoetin alfa in only 1 patient.

\section{STATISTICAL ANALYSES}

Baseline characteristics of the study patients were expressed as percentages for categorical data and median and interquartile ranges for continuous variables with skewed distributions. The Kruskal-Wallis test and onefactor analysis of variance were used to compare mean values, and chi-square test was used to evaluate differences in proportions. To examine the factors related to anemia and hemoglobin level in subjects with CKD, multivariate logistic regression analyses were performed by subdividing the data into Model 1 (adjusted for age, sex, and eGFR) and Model 2 (adjusted for the covariates in model 1 plus body mass index, history of cardiovascular disease and serum albumin). Covariates previously reported as being related to anemia in CKD patients were selected in multivariate models [8]. All statistical analyses were performed using SPSS software (version 26; IBM Corporation, Chicago, USA). A significant difference was defined as $P<0.05$.

\section{RESULTS}

After additional exclusion of patients without hemoglobin data, 1,324 participants were included in the study (Fig. 1). Of the total of 1,324 participants, 278 had primary renal disease, 412 had hypertensive nephropathy, 217 had diabetic nephropathy, and 417 had other nephropathies. The patients' baseline characteristics according to underlying renal disease are shown in Table 1. Hemoglobin levels were lower in patients with diabetic nephropathy than in the other groups.

Fig. 2 shows the distribution of hemoglobin level according to CKD stage. The percentage of patients with a hemoglobin level of $<11 \mathrm{~g} / \mathrm{dL}$ increased with $\mathrm{CKD}$ stage, with values of $7.4 \%, 17.3 \%, 31.4 \%$, and $66.7 \%$ in stage G3a, G3b, G4, and G5 CKD, respectively. Distribution of hemoglobin level also differed according to the

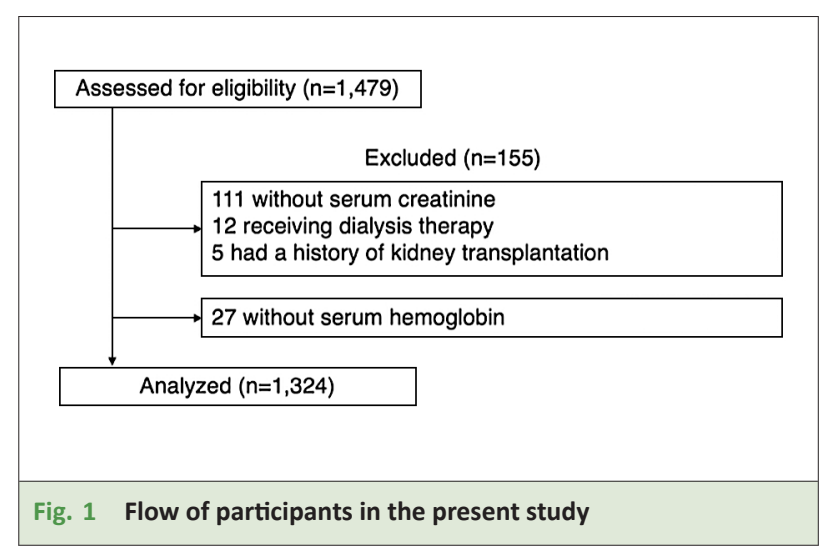




\begin{tabular}{|c|c|c|c|c|c|c|}
\hline & Total & $\begin{array}{c}\text { Primary renal } \\
\text { disease }\end{array}$ & $\begin{array}{l}\text { Hypertensive } \\
\text { nephropathy }\end{array}$ & $\begin{array}{c}\text { Diabetic } \\
\text { nephropathy }\end{array}$ & Others & $\begin{array}{l}P \text { for } \\
\text { trend }\end{array}$ \\
\hline Number of patients & 1,324 & 278 & 412 & 217 & 417 & \\
\hline Age (years) & $66.0(57.0-75.0)$ & $59.0(48.0-66.0)$ & $70.0(62.0-78.0)$ & $66.0(57.5-73.0)$ & $66.0(56.0-74.0)$ & $<0.001$ \\
\hline Age $\geq 75$ years $(\%)$ & 25.8 & 12.2 & 38.1 & 22.1 & 24.7 & $<0.001$ \\
\hline Sex (male) (\%) & 56.2 & 58.3 & 62.9 & 56.7 & 48 & $<0.001$ \\
\hline Smoking history (\%) & 52.7 & 47.8 & 58.9 & 56.7 & 47.6 & 0.003 \\
\hline Cardiovascular disease (\%) & 30.1 & 11.2 & 40.3 & 34.6 & 30.5 & $<0.001$ \\
\hline Diabetes (\%) & 48.3 & 19.8 & 46.1 & 100 & 42.4 & $<0.001$ \\
\hline Hypertension (\%) & 85.1 & 86 & 94.9 & 88.5 & 73.1 & $<0.001$ \\
\hline Dyslipidemia (\%) & 66.5 & 61.2 & 70.1 & 72.4 & 63.5 & $<0.001$ \\
\hline Body mass index $\left(\mathrm{kg} / \mathrm{m}^{2}\right)$ & $24.2(21.8-27.1)$ & $23.8(21.5-26.1)$ & $24.7(22.2-27.2)$ & $24.9(22.6-27.9)$ & $23.7(21.1-26.8)$ & $<0.001$ \\
\hline Systolic blood pressure (mmHg) & $131(119-143)$ & $126(117-136)$ & $132(121-145)$ & $136(124-150)$ & $129(117-142)$ & $<0.001$ \\
\hline Diastolic blood pressure (mmHg) & $76(68-83)$ & $78(71-85)$ & $75(68-83)$ & $76(66-83)$ & $74(66-83)$ & 0.002 \\
\hline eGFR $\left(\mathrm{mL} / \mathrm{min} / 1.73 \mathrm{~m}^{2}\right)$ & $52.0(37.8-63.8)$ & $54.8(41.7-69.4)$ & $51.2(39.1-58.9)$ & $48.8(32.2-68.9)$ & $51.5(38.5-64.6)$ & 0.022 \\
\hline Hemoglobin (g/dL) & $13.0(11.7-14.2)$ & $13.2(12.0-14.3)$ & $13.2(11.9-14.4)$ & $12.6(11.1-13.9)$ & $12.8(11.5-13.9)$ & $<0.001$ \\
\hline Ferritin (ng/dL) & $85(41-173)$ & $92(42-163)$ & $88(42-174)$ & $71(39-161)$ & $87(40-194)$ & 0.735 \\
\hline TSAT (\%) & $27.1(18.4-35.0)$ & $31.4(20.0-38.8)$ & $26.3(18.6-33.6)$ & $26.3(17.5-33.8)$ & $25.9(17.2-35.0)$ & 0.083 \\
\hline Serum albumin (g/dL) & $4.0(3.7-4.2)$ & $3.9(3.7-4.2)$ & $4.0(3.8-4.2)$ & $3.8(3.4-4.1)$ & $4.0(3.6-4.2)$ & $<0.001$ \\
\hline Proteinuria (g/gCr) & $0.21(0.03-0.85)$ & $0.33(0.10-0.86)$ & $0.09(0.00-0.40)$ & $0.82(0.20-2.80)$ & $0.10(0.00-0.40)$ & $<0.001$ \\
\hline \multicolumn{7}{|l|}{ ESA therapy (\%) } \\
\hline $\mathrm{eGFR}<45 \mathrm{~mL} / \mathrm{min} / 1.73 \mathrm{~m}^{2}$ & 19.9 & 17.4 & 10.8 & 28.7 & 24.3 & 0.003 \\
\hline$\geq 45 \mathrm{~mL} / \mathrm{min} / 1.73 \mathrm{~m}^{2}$ & 0.5 & 0.5 & 0.4 & 0.0 & 0.7 & 0.779 \\
\hline \multicolumn{7}{|l|}{ Iron therapy (\%) } \\
\hline $\mathrm{eGFR}<45 \mathrm{~mL} / \mathrm{min} / 1.73 \mathrm{~m}^{2}$ & 12.8 & 12.8 & 9.4 & 18.1 & 12.8 & 0.282 \\
\hline$\geq 45 \mathrm{~mL} / \mathrm{min} / 1.73 \mathrm{~m}^{2}$ & 4.1 & 4.7 & 2.9 & 4.1 & 4.8 & 0.684 \\
\hline Proved by renal biopsy (\%) & 25.4 & 88.8 & 3.4 & 2.8 & 16.5 & $<0.001$ \\
\hline
\end{tabular}

underlying renal disease, with the percentage of patients with a hemoglobin level of $<11 \mathrm{~g} / \mathrm{dL}$ being higher in diabetic nephropathy subjects than in other subjects (Fig. 3).

Of the 1,324 subjects, 97 (7.3\%) were treated with ESAs. The mean hemoglobin level was $10.4 \pm 1.0 \mathrm{~g} / \mathrm{dL}$ among subjects on ESA therapy, and the achievement rate of a target hemoglobin level of $\geq 11 \mathrm{~g} / \mathrm{dL}$ was $30.1 \%$. Table 2 shows the distribution of patients treated with ESA according to hemoglobin level and CKD stage. The percentage of patients receiving ESA therapy increased with CKD stage, with $0.8 \%, 8.0 \%, 23.6 \%$ and $74.5 \%$ of patients in stage G3a, G3b, G4 and G5 CKD, respectively, receiving ESA therapy. None of the patients with CKD stage G1 and G2 were being treated with ESAs. Among patients with a hemoglobin level of $<11 \mathrm{~g} / \mathrm{dL}$, the percentage of patients receiving ESA therapy was $42.9 \%$ (69 of 161) among those with stage G3a to G5 CKD, and $61.5 \%$ (48 of 78) among patients with stage G4 to G5 CKD.

Table 3 shows the distribution of patients receiving iron therapy according to iron deficiency categories. Of the 1,324 subjects, 95 (7.2\%) were on iron therapy. 

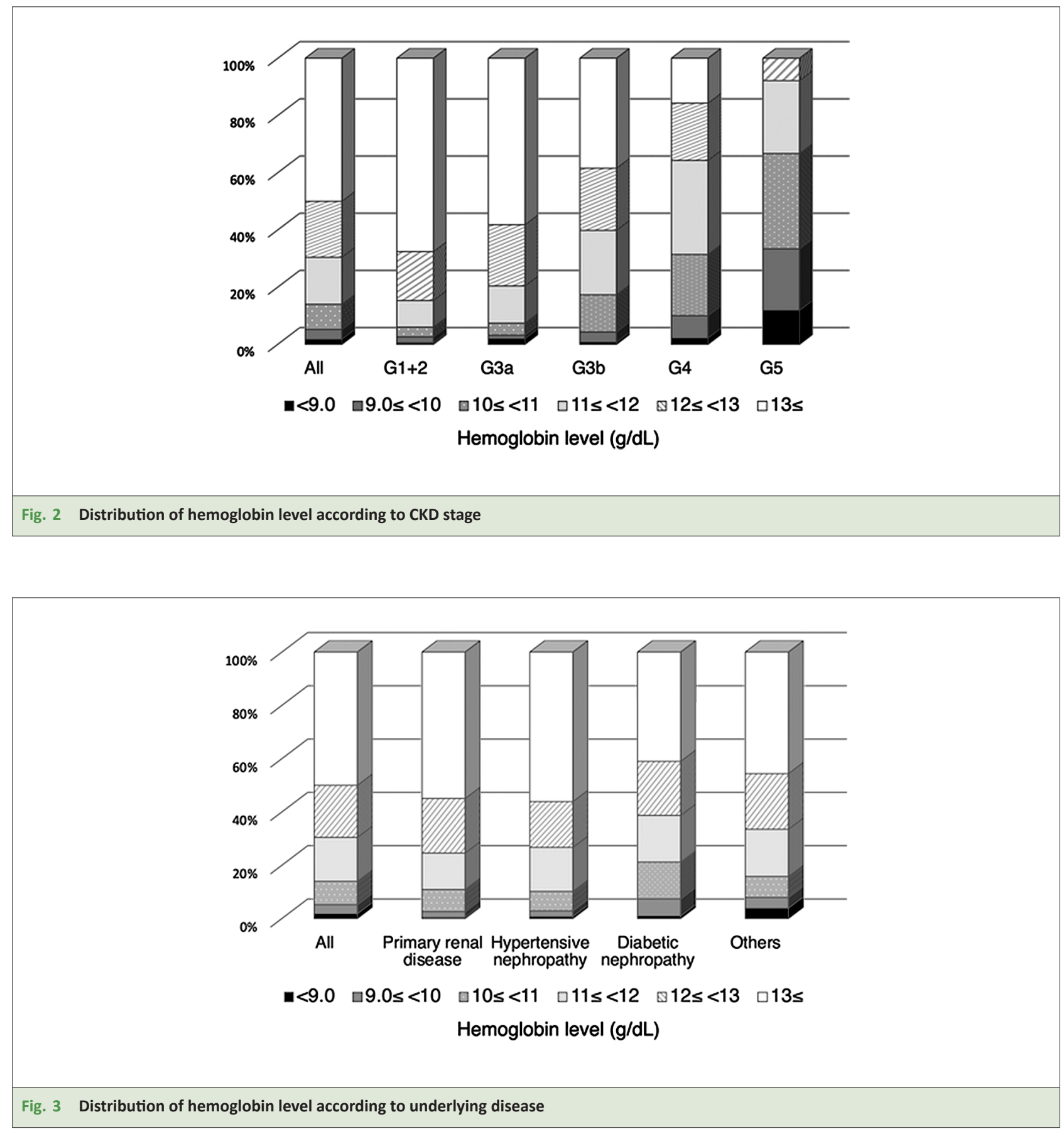

However, the percentage of patients receiving iron therapy was only $18.4 \%$ even in anemia patients with absolute iron deficiency (ferritin $<100 \mathrm{ng} / \mathrm{dL}$ and TSAT <20\%).

Fig. 4 shows the prevalence of anemia according to CKD stage and underlying renal disease. The prevalence of anemia, defined as a hemoglobin level of $<11 \mathrm{~g} / \mathrm{dL}$ or receiving ESA therapy, was $16.0 \%$ (212 of 1,324), the prevalence increasing significantly with CKD stage $(6.0 \%$, $7.4 \%, 19.2 \%, 41.4 \%$, and $84.3 \%$ in stages G1 + 2, G3a, G3b, G4, and G5, respectively, $P<0.001)$. Additionally, the prevalence of anemia was significantly higher in dia- betic nephropathy than primary renal disease and hypertensive nephropathy $(12.9 \%, 10.9 \%$, and $25.3 \%$, respectively, $P<0.001)$, this observation holding true for all stages of CKD.

Logistic regression analysis showed that anemia was independently related to the underlying renal disease, with a significant difference between diabetic nephropathy and primary renal disease (odds ratio 2.15; 95\% confidence interval, 1.16-3.99, $P=0.015$ ) (Table 4). A significant difference between diabetic nephropathy and primary renal disease was also observed with regard to a 


\begin{tabular}{|c|c|c|c|c|c|c|}
\hline Hemoglobin (g/dL) & & G3a & G3b & G4 & G5 & Total \\
\hline \multirow{2}{*}{$<10$} & $\mathrm{n}$ & $2 / 15$ & $8 / 12$ & $7 / 14$ & $16 / 17$ & $33 / 58$ \\
\hline & (\%) & 13.3 & 66.7 & 50.0 & 94.1 & 56.9 \\
\hline \multirow{2}{*}{$>10-<11$} & $\mathrm{n}$ & $2 / 20$ & $9 / 36$ & $12 / 30$ & $13 / 17$ & $36 / 103$ \\
\hline & $(\%)$ & 10.0 & 25.0 & 40.0 & 76.5 & 35.0 \\
\hline \multirow{2}{*}{$>11$} & $\mathrm{n}$ & $0 / 436$ & $5 / 228$ & $14 / 96$ & $9 / 17$ & $28 / 777$ \\
\hline & (\%) & 0.0 & 2.2 & 14.6 & 52.9 & 3.6 \\
\hline \multirow{2}{*}{ Total } & $\mathrm{n}$ & $4 / 471$ & $22 / 276$ & $33 / 140$ & $38 / 51$ & $97 / 938$ \\
\hline & $(\%)$ & 0.8 & 8.0 & 23.6 & 74.5 & 10.3 \\
\hline
\end{tabular}

\begin{tabular}{|c|c|c|c|c|c|c|c|}
\hline & & $\begin{array}{c}\text { Ferritin }<100 \mathrm{ng} / \mathrm{dL} \\
\text { and TSAT }<20 \%\end{array}$ & $\begin{array}{c}\text { Ferritin } \geq 100 \mathrm{ng} / \mathrm{dL} \\
\text { and TSAT }<20 \%\end{array}$ & $\begin{array}{l}\text { Ferritin }<100 \mathrm{ng} / \mathrm{dL} \\
\text { and TSAT } \geq 20 \%\end{array}$ & $\begin{array}{c}\text { Ferritin } \geq 100 \mathrm{ng} / \mathrm{dL} \\
\text { and TSAT } \geq 20 \%\end{array}$ & $\begin{array}{c}\text { Missing data of } \\
\text { ferritin and/or TSAT }\end{array}$ & Total \\
\hline \multirow{2}{*}{ With anemia } & $\mathrm{n}$ & $7 / 38$ & $4 / 14$ & $17 / 48$ & $11 / 69$ & $1 / 43$ & $40 / 212$ \\
\hline & (\%) & 18.4 & 28.6 & 35.4 & 15.9 & 2.3 & 18.9 \\
\hline \multirow{2}{*}{ Without anemia } & $\mathrm{n}$ & $12 / 69$ & $2 / 23$ & $17 / 119$ & $14 / 117$ & $10 / 784$ & $55 / 1112$ \\
\hline & $(\%)$ & 17.4 & 8.7 & 14.3 & 12 & 1.3 & 4.9 \\
\hline \multirow{2}{*}{ Total } & $\mathrm{n}$ & $19 / 107$ & $6 / 37$ & $34 / 167$ & $25 / 186$ & $11 / 827$ & $95 / 1324$ \\
\hline & (\%) & 17.8 & 16.2 & 20.4 & 13.4 & 1.3 & 7.2 \\
\hline
\end{tabular}

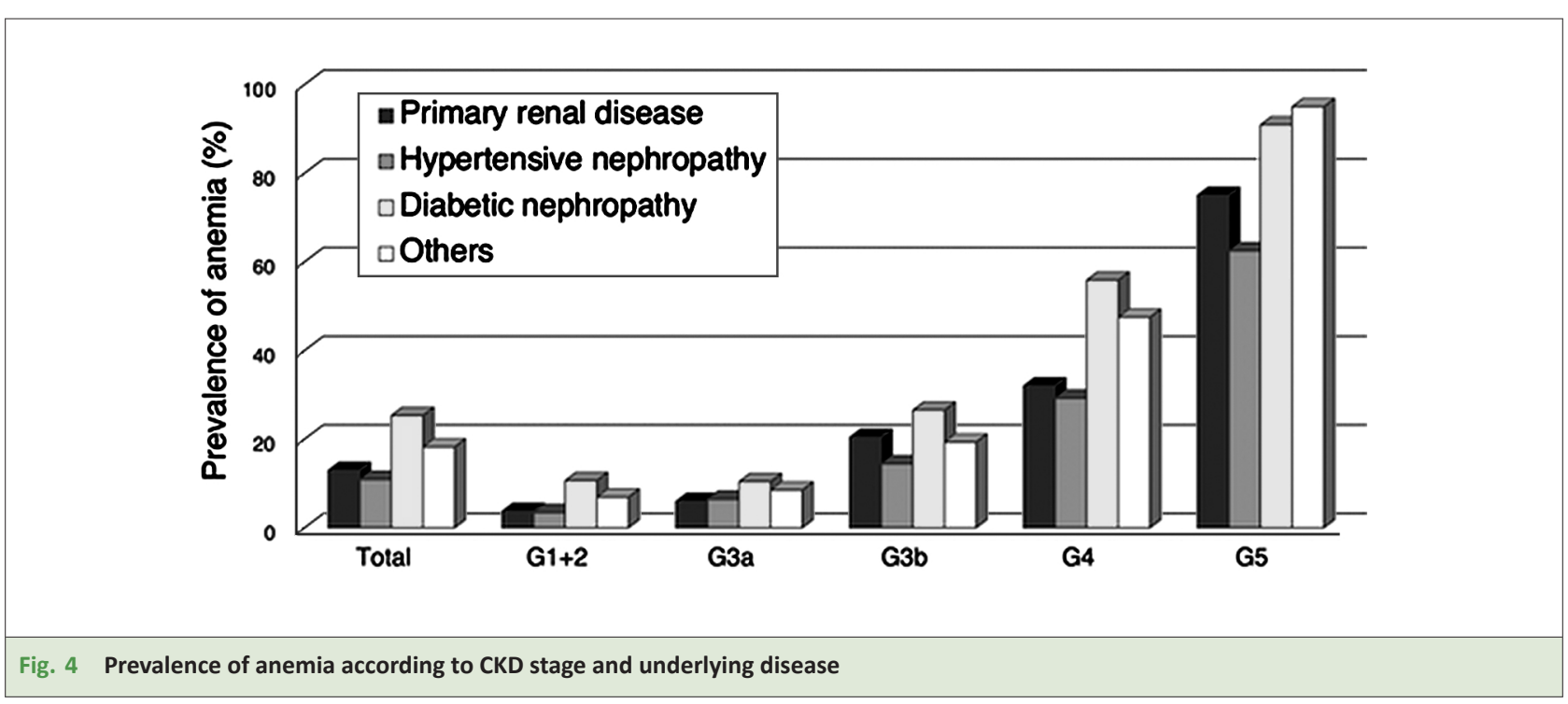

hemoglobin level of $<11 \mathrm{~g} / \mathrm{dL}$ (odds ratio 1.99; 95\% confidence interval 1.06-3.74, $P=0.032$ ).

\section{DISCUSSION}

The present study showed the inadequate management status of anemia even in pre-dialysis CKD patients under a nephrologist's care, including with current treatment 


\begin{tabular}{|c|c|c|c|c|c|c|}
\hline & \multicolumn{2}{|c|}{ Unadjusted } & \multicolumn{2}{|c|}{ Model 1} & \multicolumn{2}{|c|}{ Model 2} \\
\hline & OR $(95 \%$ CI $)$ & $P$ value & OR $(95 \%$ CI $)$ & $P$ value & OR $(95 \%$ CI $)$ & $P$ value \\
\hline \multicolumn{7}{|l|}{ Anemia } \\
\hline Primary renal disease & Reference & & Reference & & Reference & \\
\hline Hypertensive nephropathy & $0.82(0.52-1.32)$ & 0.418 & $0.71(0.42-1.20)$ & 0.206 & $1.22(0.68-2.20)$ & 0.510 \\
\hline Diabetic nephropathy & $2.28(1.43-3.63)$ & 0.001 & $1.90(1.12-3.20)$ & 0.017 & $2.15(1.16-3.99)$ & 0.015 \\
\hline Others & $1.50(0.98-2.30)$ & 0.065 & $1.28(0.79-2.07)$ & 0.314 & $1.56(0.91-2.69)$ & 0.108 \\
\hline \multicolumn{7}{|l|}{ Hemoglobin <11 g/dL } \\
\hline Primary renal disease & Reference & & Reference & & Reference & \\
\hline Hypertensive nephropathy & $0.94(0.57-1.54)$ & 0.801 & $0.82(0.48-1.40)$ & 0.461 & $1.38(0.75-2.54)$ & 0.294 \\
\hline Diabetic nephropathy & $2.22(1.35-3.67)$ & 0.002 & $1.82(1.06-3.13)$ & 0.031 & $1.99(1.06-3.74)$ & 0.032 \\
\hline Others & $1.55(0.98-2.47)$ & 0.061 & $1.30(0.79-2.14)$ & 0.299 & $1.54(0.88-2.69)$ & 0.132 \\
\hline
\end{tabular}

for anemia using long-acting ESAs, such as darbepoetin alfa or epoetin beta pegol. The prevalence of anemia, defined as a hemoglobin level of $<11 \mathrm{~g} / \mathrm{dL}$ or receiving ESA therapy, increased with the progression of CKD stage, and was higher in patients with diabetic nephropathy than those with other nephropathies. Diabetic nephropathy had an independent association with anemia after adjustment for covariates in multivariate logistic regression analysis.

Our results suggested that the currently followed intervention strategy for renal anemia is not necessarily sufficient in these patients. Treatment guidelines for renal anemia recommend a target hemoglobin of 11-13 g/dL for pre-dialysis patients with CKD in Japan [12, 13]. However, subjects on ESA therapy accounted for only $42.9 \%$ and $56.9 \%$ of those with a hemoglobin $<11 \mathrm{~g} / \mathrm{dL}$ and $<10 \mathrm{~g} / \mathrm{dL}$, respectively, among CKD stage G3a to G5 patients in the present study. The mean hemoglobin level was $10.4 \pm 1.0 \mathrm{~g} / \mathrm{dL}$ among subjects on ESA therapy and the achievement rate of a target hemoglobin concentration of $\geq 11 \mathrm{~g} / \mathrm{dL}$, recommended in Japanese guidelines, was only $30.1 \%$.

The levels of both ferritin and TSAT were low in the present study, and the percentage of patients with supplemental iron therapy was quite low even in anemia patients with absolute iron deficiency. These results suggest that interventions for renal anemia with ESA as well as iron supplements according to the recommendations of Japanese guidelines are not necessarily sufficient even among CKD patients under a nephrologist's care.

However, kidney disease improving global outcomes (KDIGO) clinical practice guidelines for anemia in CKD recommend that ESA therapy should not be initiated for patients with a hemoglobin concentration $\geq 10 \mathrm{~g} / \mathrm{dL}$, and ESAs should not be used to maintain the hemoglobin concentration at $\geq 11.5 \mathrm{~g} / \mathrm{dL}$ in adults with non-dialysis CKD [14]. Therefore, anemia control was not necessarily insufficient in these patients according to the recommendations of the international KDIGO guideline, although the discrepancies between Japanese and KDIGO guidelines might have affected the clinical practice strategy for renal anemia and the target hemoglobin concentration in CKD patients in the present study.

A previous cohort study also described failure to achieve the target hemoglobin level. A previous study using baseline data of the CKD-JAC study showed that the percentage of patients on ESA therapy was $32.4 \%$ among those with a hemoglobin concentration of $<11 \mathrm{~g} / \mathrm{dL}$, and the achievement rate of a target hemoglobin concentration of $\geq 11 \mathrm{~g} / \mathrm{dL}$ among patients on ESA therapy was $30.1 \%$ in CKD stage G3a to G5 patients under treatment for renal anemia with epoetin alfa and beta [8]. As a low achievement rate of target hemoglobin was prominent among patients only receiving ESAs infrequently, the difficulty in maintaining dosing intervals in an outpatient setting was thought to be one of the reasons for the poor management status of anemia in this study. Although the percentage of patients on ESA therapy among those with 
a hemoglobin of $<11 \mathrm{~g} / \mathrm{dL}$ was higher in the present study than in the CKD-JAC study, the achievement rate of the target hemoglobin concentration of $\geq 11 \mathrm{~g} / \mathrm{dL}$ among patients on ESA therapy was not different in the present versus previous studies even under the current treatment regimen for anemia, including long-acting ESAs.

It is possible that difficulty in achieving the target hemoglobin level was not due to insufficient anemia care by the nephrologists, but due to ESA hyporesponsiveness in some patients. As the management status of renal anemia was not improved even in patients on long-acting ESAs, the potential causes of the poor management status of renal anemia in the present study, which could be explained by ESA hyporesponsiveness caused by malnutrition or inflammation, anxiety in using high-dose ESAs, iron insufficiency, bone marrow disease, high cost, need for injections for ESA treatment, or clinical inertia, should be investigated in detail in the future. In any case, our results suggest that current medications, including long-acting ESAs, might not necessarily be sufficient for achieving this target range of hemoglobin.

The prevalence of anemia differed according to the underlying renal disease, and was higher in diabetic nephropathy than in other nephropathies in the present study. Previous reports suggested that anemia associated with erythropoietin (EPO) deficiency can occur early in diabetic nephropathy, before the onset of advanced renal failure, but does not normally occur in non-diabetic renal disease of similar severity [15]. The recent findings of improvement in anemia with sodium-glucose cotransporter 2 (SGLT2) inhibitors have suggested that tubulointerstitial hypoxia caused by the high oxygen requirement of proximal tubules due to excessive glucose reabsorption could exacerbate anemia in diabetes [16]. Although this mechanism could be one of the reasons for the higher prevalence of anemia, as well as poor renal outcomes, in diabetic nephropathy, the relationship between glucose reabsorption, interstitial damage and EPO production should be closely investigated both in basic experiments and clinical studies in the future.

The present study has several limitations. First, single measurements of hemoglobin and serum creatinine might have led to some misclassification of CKD and the diagnosis of anemia. Second, since this study was a crosssectional study, it is necessary to examine the relationships between hemoglobin level, management status of anemia with ESA treatment, CKD progression, cardiovascular disease and mortality according to underlying renal disease in these patients. Third, since data of ESA dosage used by the patients was not available in the present study, hyporesponsiveness or resistance to ESA, which could have affected the management status of anemia, was not investigated. In future, whether the inadequate management status of renal anemia was caused by insufficient use of ESA or hyporesponsiveness to ESA should be investigated in detail. Additionally, we did not have data on serum hepcidin levels, which would affect both hyporesponsiveness to ESA and the management status of anemia in CKD patients. These limitations of the present study need to be addressed in future studies.

\section{CONCLUSIONS}

In the present study, insufficient management status of renal anemia was observed even in patients under a nephrologist's care, including those on current treatment with long-acting ESAs, in Japanese patients with predialysis CKD. These results might indicate that the current medication strategy of iron supplements and ESA for renal anemia is insufficient for achievement of the recommended hemoglobin level in these patients. In future, whether the inadequate management status of renal anemia relates to poor outcomes in pre-dialysis patients with CKD should be investigated in a longitudinal survey of sufficient size and with better statistical methods.

\section{CONFLICT OF INTEREST}

The authors declare no conflicts of interest in relation to this work.

\section{ACKNOWLEDGMENTS}

The authors would like to thank Ayumi Kanno for her assistance in data collection.
1. Go AS, Chertow GM, Fan D, McCulloch CE, Hsu CY. Chronic kidney disease and the risks of death, cardiovascular events, and hospitalization. N Engl J Med 2004;351:1296-305. 2. Keith DS, Nichols GA, Gullion CM, Brown JB, Smith DH. Longitudinal follow-up and outcomes among a population with chronic kidney disease in a large managed care organ- ization. Arch Intern Med 2004;164:659-63. 3. Imai $\mathrm{E}$, Horio $\mathrm{M}$, Watanabe $\mathrm{T}$, Iseki $\mathrm{K}$, Yamagata K, Hara S, et al. Prevalence of chronic kidney disease in the Japanese general population. Clin Exp Nephrol 2009;13:621-30. 4. Masakane I, Nakai S, Ogata S, Kimata N, Hanafusa N, Hamano T, et al. An Overview of Regular Dialysis Treatment in Japan (As of
31 December 2013). Ther Apher Dial 2015; 19:540-74.

5. Yamagata K, Ishida K, Sairenchi T, Takahashi $\mathrm{H}$, Ohba S, Shiigai T, et al. Risk factors for chronic kidney disease in a community-based population: a 10-year follow-up study. Kidney Int 2007;71:159-66.

6. Stauffer ME, Fan T. Prevalence of anemia 
in chronic kidney disease in the United States. PLoS One 2014;9:e84943.

7. Go AS, Yang J, Ackerson LM, Lepper K, Robbins S, Massie BM, et al. Hemoglobin level, chronic kidney disease, and the risks of death and hospitalization in adults with chronic heart failure: the Anemia in Chronic Heart Failure: Outcomes and Resource Utilization (ANCHOR) Study. Circulation 2006; 113:2713-23.

8. Akizawa T, Makino H, Matsuo S, Watanabe T, Imai E, Nitta $K$, et al. Management of anemia in chronic kidney disease patients: baseline findings from Chronic Kidney Disease Japan Cohort Study. Clin Exp Nephrol 2011;15:248-57.

9. Nakayama M, Sato T, Sato H, Yamaguchi Y, Obara K, Kurihara I, et al. Different clinical outcomes for cardiovascular events and mor- tality in chronic kidney disease according to underlying renal disease: the Gonryo study. Clin Exp Nephrol 2010;14:333-9.

10. Nakajima A, Tanaka K, Saito H, Iwasaki T, Oda A, Kanno M, et al. Blood pressure control in chronic kidney disease according to underlying renal disease: the Fukushima CKD cohort. Clin Exp Nephrol 2019 Dec.

11. Matsuo S, Imai E, Horio M, Yasuda Y, Tomita K, Nitta K, et al. Revised equations for estimated GFR from serum creatinine in Japan. Am J Kidney Dis 2009;53:982-92.

12. Yamamoto H, Nishi S, Tomo T, Masakane I, Saito K, Nangaku M, et al. 2015 Japanese Society for Dialysis Therapy: Guidelines for Renal Anemia in Chronic Kidney Disease. Renal Replacement Therapy 2017;3:36.

13. Nephrology JSo. Essential points from
Evidence-based Clinical Practice Guidelines for Chronic Kidney Disease 2018. Clin Exp Nephrol 2019;23:1-15.

14. Drueke TB, Parfrey PS. Summary of the KDIGO guideline on anemia and comment: reading between the (guide)line(s). Kidney Int 2012;82:952-60.

15. Bosman DR, Winkler AS, Marsden JT, Macdougall IC, Watkins PJ. Anemia with erythropoietin deficiency occurs early in diabetic nephropathy. Diabetes Care 2001;24: 495-9.

16. Sano M, Takei M, Shiraishi Y, Suzuki Y. Increased Hematocrit During SodiumGlucose Cotransporter 2 Inhibitor Therapy Indicates Recovery of Tubulointerstitial Function in Diabetic Kidneys. J Clin Med Res 2016;8:844-7. 